Intracranial Stereotactic Radiosurgery. 2009. By L. Dade Lunsford, Jason P. Sheehan. Published by Thieme Medical Publishers. 182 pages. $\mathrm{C} \$ 185$ approx.

\section{Rated trotstror}

This is an excellent book, but it is what one would expect from the editors who are leaders in the field of stereotactic radiosurgery. Although small (182 pages), it is a comprehensive reference for anybody who deals with patients with intracranial pathology which might be suitable to stereotactic radiosurgery treatment.

The first four chapters overview development, radiobiology, tumor tissue response to stereotactic radiosurgery, and general principles of the CyberKnife. The following chapters discuss indications, outcome, and complications for specific pathologies which are treated with stereotactic radiosurgery. Of interest, there is a chapter about stereotactic radiosurgery procedures for ocular disorders. The last four chapters deal with radiosurgical treatment of malignant brain tumors. This book provides the latest information on the subject with references up to 2007 .

The only critique I have is that some authors reported only their own (although very extensive) experience without discussing data from other treatment centers. Also, there is very little about other forms of stereotactic radiosurgery like LINAC and proton beam treatment. I think that should have been included for completeness of the information.

I would highly recommend this book to anyone who deals with intracranial (and ocular) pathology, which may be amenable to stereotactic radiosurgery treatment. It serves as an excellent resource for physicians to provide their patients with the necessary information about this treatment and as such it can be used not only by Neurosurgeons, but also Neurologists and other physicians who may refer patients for intracranial stereotactic radiosurgery. It is also an excellent source of necessary information for neurosurgical residents in training.

\section{Aleksander M. Vitali Saskatoon, Saskatchewan, Canada}

Animal Models of Epilepsy: Methods and innovations. 2009. Edited by Scott C. Baraban. Published by Humana Press. 272 pages. C\$150 approx.

\section{Rated UNAVAILABLE}

As a clinical epileptologist interested in fundamental aspects of epilepsy, I obtained a thorough update on the several frontiers of current epilepsy research from this lucid volume. Basic scientists will profit from these 14 distinct and succinct reviews that also treat methodology and pitfalls in detail. Thus, this work is recommended for basic science and clinical epileptologists as well as students of these disciplines. Comparison with Experimental Models of Epilepsy (1972) edited by $\mathrm{D}$. Purpura et al discloses the considerable progress in models, topics confronted and methodology over the past 37 years. However, electrophysiology remains at the forefront!

Chapter 1: The nematode, caennorhabditis elegans, as an emerging model for investigating epilepsy. Locke CJ, Caldwell KA, Caldwell GA.

Because of their small size and much commonality with mammalian neuro-physiology, nematodes, specifically C. Elegans can serve as a model of an entire nervous system. A prolific self fertilizer, 300 offspring per parent may appear; adulthood is attained within three days! This species shares many cellular and molecular pathways with mammals, including humans. Of its $\sim 1000$ somatic cells, $\sim 300$ are neurons, organized into the components of which the "nerve ring" appears to roughly correspond to a brain. Human neurotransmitters including glutamate, GABA, acetylcholine, dopamine and serotonin have been found in C.Elegans. GABA mediates foraging behaviour; eliminating GABA alters motor movements of foraging. Patterns of locomotion alter depending on the balance of excitatory and inhibitory transmission at neuromuscular junctions. Thus, behavioural assays enable isolation of genes affecting neuronal excitability. Electrophysiological data have been difficult to obtain, but optical imaging techniques have provided behavioural -physiological correlations.

Chapter 2: The genetics and molecular biology of seizure susceptibility in drosophila. Song J, Tanouye MA.

Surprising similarities relate Drosophilia (fruit fly) to mammalian nervous systems: voltage -gated and ligand-gated signaling molecules including: sodium, potassium, and calcium channels as well as acetylcholine, glutamate, and GABA transmitters and receptors. Thus Drosophila has the potential to model several human central nervous system disorders. A set of seizure-sensitive Drosophila mutants has been developed that share some features of human epilepsies. That two mutations may create a phenotype that differs from that resulting from either mutation alone suggests a basis for variability within given human epilepsy syndromes. Easily identifiable seizure suppressor mutations abundantly appear in Drosophila. These were initially disclosed through a reverse genetics approach, i.e. seeking mutants that would suppress seizure-like behaviour in bang-sensitive (BS) and other Drosophila mutants. Moreover, mutation of a gene encoding a gap junction protein impairs electrical transmission and elevates seizure threshold in this species. These data may well lead to effective anti-epileptic drug (AED) development. These authors propose that an effective way to discover unexpected seizure suppressor genes is using an unbiased "forward genetics" approach that is detailed in their chapter.

Chapter 3: The albino xenopus laevis tadpole as a novel model of developmental seizures. Hewapathirane DS, Haas $K$.

Whether early life seizures cause later comorbidities such as seizures, cognitive impairment or behavioural abnormalities or whether early seizures and later comorbidities share a common pathogenesis remains uncertain. These authors propose use of Xenopus laevis tadpole to answer this question. This organism is widely used to study early brain development and its neuronal physiology shares many aspects with mammalian species. These 
tadpoles exhibit free-swimming by age three days and most studies are performed at age five days. Extracellular field electrodes can record seizure activity while the tadpole is immobilized by bath application of a reversible paralytic agent, eliminating the confounding effect of anaesthetic agents. Calcium-sensitive fluorescent indicators can monitor seizure activity throughout the intact brain with single-cell resolution. After loading neurons with calcium sensitive dyes, fluorescence microscopy can measure changes in cytoplasmic calcium concentration during seizures. One awaits the application of this technique to the above-stated question.

Chapter 4: Zebrafish as a simple vertebrate organism for epilepsy research. Baraban $S C$.

This review explores underlying mechanisms of seizure generation in developing zebrafish. As with organisms discussed in earlier chapters, such research benefits from the facility of maintaining large colonies, short generation times and hundreds of offspring. The optical transparency of its larvae is a technical advantage. As with earlier-discussed species, mammalian neurotransmitters are present: glutamine, GABA, glycine, serotonin and catecholamines. Pentylenetetrazol (PTZ) application evokes clustered diffuse (forebrain, midbrain, hindbrain) epileptiform discharges as a burst suppression pattern resembling, for this author, that seen in pediatric epilepsy syndromes. These apparently modify as the zebrafish brain matures. As with other models, optical imaging of fluctuations in fluorescence of calcium sensitive dyes (method in Chapter 9) can also portray ictal activity. Such PTZ-induced seizures in zebrafish offer a rapid method to screen large numbers of possible AEDs.

Using zebrafish as a model of epilepsy in the tuberous sclerosis complex (TSC), this author found several TSC genes in this species. Disrupting such gene function with antisense nucleotides produced frequent "large-amplitude" epileptiform discharges. Importantly, such occurred in the absence of tubers or other TSC pathology. This implies that reduced hamartin-tuberin expression alters neuronal or synaptic function to create excessive excitation, thus seizure activity.

Chapter 5: Modeling tuberous sclerosis complex: brain development and hyperexcitability. Ess $K C$.

Although inheritable as an autosomal dominant disorder, spontaneous mutations of either TSC I or TSC 2 gene may produce the full spectrum of TSC. Identification of their homologues in several species such as mouse, rat, and Drosophila allow examination of the TSC signaling pathway. From such knowledge mTOR kinase and its downstream signaling cascade has emerged as a potential therapeutic target; rapamycin can effectively regulate the mTOR pathway. Rapamycin in the mouse model of TSC has prolonged survival and controlled its seizures. An additional research avenue is "knock in": mouse experiments in which normal TSC 1 or TSC 2 genes are replaced by alleles containing disease-causing mutations.

Chapter 6: BK potassium channel mutations affecting neuronal function and epilepsy. Petrik $D$, Chen $Q H$, Brenner $R$.

A large conductance voltage and calcium-activated potassium channel (BK channel) gene has been identified in humans and mice that paradoxically increases excitability and seizures. BK channels can "sharpen" action potentials by removing inactivation of sodium channels while deactivating quickly to allow subsequent action potentials. (The possibility remains that BK channels could also reduce excitability of interneurones and thereby augment excitability.) The $\beta 4 \mathrm{BK}$ channel subunit, an inhibitor of BK channel activation, is abundant in the CNS; $\beta 4$ knockout mice exhibit spontaneous nonconvulsive temporal lobe-like seizures. Moreover, $\beta 4$ is down- regulated in a rat model of electrically-induced epilepsy. However, the authors indicate that further studies of the complex interplay between BK channels and other ionic currents will help distinguish between adaptive and maladaptive changes affecting epileptogenesis.

Chapter 7: Mouse models of benign familial neonatal convulsions (BFNC): mutations in KCNQ $(K v 7)$ genes. Singh NA, Otto JF, Leppert $M F$, White $H S$, Wilcox $K S$.

Genetic linkage analyses have disclosed that mutations of either KCNQ2 gene on chromosome $20 \mathrm{q}$ or (less frequently) KCNQ3 gene on chromosome $8 \mathrm{q}$ produce BFNC. These genes, along with KCNQ5, encode respective subunits comprising the $\mathbf{M}$ type potassium channel. Such subunit expression studies have been conducted in the Xenopus laevis oocyte, the Chinese hamster ovary cell, and the human embryonic kidney cell. The $M$ potassium current is tonically active at resting membrane potential, activates in response to membrane depolarization, and is blocked by acetylcholine receptor activation. The authors indicate that combining whole animal behaviour with single cell biophysics solidifies the link between KCNQ2 and KCNQ3 mutations attenuating $M$ potassium current and increased seizure susceptibility.

Chapter 8: Interneuron loss as a cause of seizures: lessons from interneuron-deficient mice. Jones-Davis D, Calcagnotto M-E, Sebe $J Y$.

Many studies of epileptic patients and animal models of epilepsy have supported the association between seizures and loss of GABAergic transmission. Electrophysiological investigations of dentate granule cells of epileptic patients have disclosed a reduction in inhibitory synaptic transmission. The interneuron-deficient transgenic mice model has recently confirmed that this association is causal. This model will shed further light upon interneuron development, interneuron function, and the importance of GABAergic inhibition in preventing seizure activity. Data from the mouse models described in this chapter suggest that seizure severity may depend upon the type of inhibition and interneuron subpopulation lost.

Chapter 9: Imaging seizure propagation in vitro. Trevelyan AJ, Yuste $R$.

Intracellular recordings detail the activity of single neurons or a cluster thereof. Extracellular recordings depict function of a neuronal network but lack cellular resolution. A more comprehensive method that monitors both aspects is cellular imaging signals correlated with electrophysiological recordings. The authors of this chapter list some drawbacks of in vitro preparations including: 1) loss of long neuronal connections, but most cellular interactions are local; 2) neuromodulators such as serotonin, acetylcholine and nor-adrenaline may be modulated; and 3) cooling during slicing may depolarise cells, temporarily collapse dendritic spines and may terminate seizures. Benefits include control over experimental conditions and enhanced visualization of tissue in vitro as opposed to in vivo.

Fast network imaging of calcium transients is accomplished by loading the tissue with a calcium-sensitive dye by which activity of hundreds of neurons can be simultaneously measured with single-cell precision. Limitations of such dye labeling are indicated: asymmetrical kinetics of calcium binding, less ability to load hippocampus and thalamus than neocortex, and blurring of the signal by high frequency action potentials. However, as stated, simultaneous electrophysiological recordings allow exact correlation of electric activity with the calcium signal pattern.

The inhibitory surround, described by Prince and Wilder (1967) consists of a suppression of cortical function immediately surrounding an area of activation. This mechanism acts to contain excitation within 
a focus, i.e. restricting its spread. To preserve inhibition in a network, a zero magnesium milieu can be employed. Ictal propagation proceeds slowly in this medium, permitting a "more tractable imaging proposition." Possibly as a consequence of zero magnesium milieu, lower intracellular magnesium diminishes Mg-ATP levels resulting in a gradual reduction in GABA receptor phosphorylation thus disinhibiting the slice.

Using this method, the authors found that succeeding seizures propagate faster than the ones before. They observed rhythmic gamma-frequency neuronal depolarisations to precede the ictal wavefront by several hundred microns; rhythmicity of the advance depolarisations matched that of the ictal wavefront. However, such neurons failed to fire due to feedforward inhibition. Recruitment of neurons into the ictus begins upon failure of this inhibitory wavefront similar to that described by Matsumoto and Ajmone Marsan (1964). Chapter 10: Complexity untangled: large-scale realistic computational models in epilepsy. Morgan RJ, Soltesz I.

The authors indicate that computational modeling has struggled to balance "physiological realism with computational reality". Solutions have been to either use small networks of detailed cells or large networks of relatively simple cells. This chapter focuses on models which incorporate detailed single cells into complex large-scale physiologically realistic networks involving accurate topographies and huge numbers of synapses. For example, after an acute insult, the dentate gyrus undergoes marked structural reorganization. This provides an experimental model to study effects of such reorganization on epileptogenesis. However, the authors maintain that only computational modeling allows study of a particular network alteration in isolation.

Chapter 11: Organotypic hippocampal slice cultures as a model of limbic epileptogenesis. Bausch $S B$.

Hippocampal slice cultures, with their short latent period, can be treated promptly with a known reagent without systemic side effects creating an attractive alternative to in vivo models. Thinner than acute slices, they are more appropriate for imaging neuronal circuitry and can be maintained for longer periods. Synaptic connectivity is far more maintained in hippocampal slice cultures than acute slices. Roller tube and interface methods, those most commonly employed, are described in detail. Most such cultures are isolated from the early postnatal rat or mouse. Roller tube is the thinner of these most commonly used methods making them preferable for standard or high optical resolution imaging. The thicker interface cultures provide a better three dimensional structure, thus more appropriate for electrophysiological recordings of neuronal populations. The aforementioned short latent period facilitates effects of drugs on epileptogenesis.

Chapter 12: Seizure analysis and detection in vivo. Echauz J, Wong S, Litt $B$.

Comprehension of clinical and electrographic seizure phenomena, from interical to ictal states, is much facilitated by continuous EEG recording. This chapter details steps in accomplishing seizure detection in human and animal recording, including a set of computational "tools" found helpful in the authors' laboratories. Four steps in quantitative seizure detection are detailed: 1) data preprocessing (e.g. artefact removal, notch filtering), 2) feature extraction, 3) classifier training, and 4) validation. Details and challenges are confronted.

Chapter 13: Viral vector gene therapy for epilepsy. Foti SB, Russek SJ, Brooks-Kayal AR, McCown TJ.
Recombinant adeno-associated virus (AAV) vectors can suppress seizures by interrupting NMDA receptor function or by altering GABA receptor composition. Viral vectors based upon adenovirus (Ad), human immunodeficiency (HIV) virus, or herpes simplex virus (HSV) have also been employed for this purpose. Altering postsynaptic receptor or membrane-bound ion channel function attenuates seizure activity. Because viral vectors such as Ad, AAV, HIV-1, and HSV exhibit neurotropism, these targets are accessible. Humans with temporal lobe epilepsy and adult rodents have reduced expression of $\alpha 1$ subunit of GABAa receptors. An AAV vector, designed to express RNA that codes for GABA receptor $\alpha 1$ subunit, augmented dentate granule subunit $\alpha 1$ in rats and decreased seizure activity after status epilepticus in this model. However, two areas of caution exist: 1) specific patterns of transduction may alter the outcome of therapeutic gene expression, and 2) different promoters may alter gene expression even within the same viral vector.

Chapter 14: Neural stem cells in experimental mesial temporal lobe epilepsy. Kron MM, Parent JM.

Neuronal stem cells (NSCs) persist in the adult mammalian brain; these can be "expanded" in vitro as a source of transplantable cells for repair. Neurogenesis in the dentate gyrus is increased by status epilepticus producing abnormalities such as granule cell layer dispersion and aberrant granule cell migration to the hilar cell basal dendrites. Adult-generated dentate granule cells develop functional synapses whose electrical activity integrates into the hippocampal network; such ectopic cells are apparently hyperexcitable. However, other studies suggest that post-seizure neurogenesis may restore inhibition. In contrast, neurogenesis may decrease in chronic mesial temporal epilepsy and this decline may underlie the progressive memory impairment in this disorder. Despite evidence of decreased GABA in epileptic brain, GABA-mimetic therapies have been generally ineffective. Stem cell grafting, potentially more targetable, may more effectively control seizures. Pitfalls exist: 1) any grafting on interneurones may decrease inhibition, and 2) a critical period during neuronal differentiation may occur during which developing neurons could be recruited into epileptogenic networks. 